\title{
BMJ Open Efficacy of oral administration of cystine and theanine in patients with colorectal cancer undergoing capecitabine-based adjuvant chemotherapy after surgery: study protocol for a multi-institutional, randomised, double-blinded, placebo- controlled, phase II trial
}

\author{
Reo Hamaguchi, ${ }^{1}$ Takashi Tsuchiya, ${ }^{2}$ Go Miyata, ${ }^{3}$ Toshihiko Sato, ${ }^{4}$ \\ Kenichi Takahashi, ${ }^{5}$ Keisuke Ariyoshi, ${ }^{1,6}$ Shunsuke Oyamada, ${ }^{6}$ Satoru Iwase ${ }^{1}$
}

To cite: Hamaguchi R, Tsuchiya T, Miyata G, et al. Efficacy of oral administration of cystine and theanine in patients with colorectal cancer undergoing capecitabinebased adjuvant chemotherapy after surgery: study protocol for a multi-institutional, randomised, doubleblinded, placebo-controlled, phase II trial. BMJ Open 2018;8:e021442. doi:10.1136/ bmjopen-2017-021442

- Prepublication history and additional material for this paper are available online. To view please visit the journal online (http://dx.doi.org/10.1136/ bmjopen-2017-021442).

Received 28 December 2017 Revised 29 April 2018 Accepted 13 June 2018

Check for updates

(C) Author(s) (or their employer(s)) 2018. Re-use permitted under CC BY-NC. No commercial re-use. See rights and permissions. Published by BMJ.

For numbered affiliations see end of article.

Correspondence to Dr Reo Hamaguchi; reo-h@nifty.com

\begin{abstract}
Introduction Although adjuvant capecitabine therapy for patients with colorectal cancer after surgery often causes adverse events (AEs), such as diarrhoea, stomatitis, anorexia and hand-foot syndrome (HFS), there are no standard prevention therapies. Cystine and theanine were reported to attenuate some chemotherapy-associated AEs, and are also expected to attenuate the AEs caused by capecitabine treatment. Therefore, our present study aimed to determine the safety and efficacy of cystine/ theanine therapy in patients with colorectal cancer undergoing capecitabine-based adjuvant chemotherapy after surgery.

Methods and analysis A multi-institutional, prospective,
\end{abstract} randomised, double-blinded, placebo-controlled, phase II trial is being planned. Patients with colorectal cancer treated with capecitabine as an adjuvant chemotherapy will be randomised into either the cystine/theanine group $(n=50)$ or placebo group ( $n=50$ ). Data will be collected during four courses of capecitabine therapy. The primary endpoint will be incidence rate of diarrhoea of grade 1 or higher in accordance with the Common Terminology Criteria for AEs (CTCAE) v.4.0, Japanese Clinical Oncology Group (JCOG) version. The secondary endpoints are incidence rates of other AEs (CTCAE v.4.0-JCOG), scores of the Japanese version of the European Organisation for Research and Treatment of Cancer Quality of Life Questionnaire module for all patients with cancer (QLQ-C30) and for patients with colorectal cancer (QLQ-CR29), incidence rate of HFS according to the HFS grading scale, protocol adherence, completion rate of four courses of capecitabine therapy and the proportion of completion without delay or dose reduction, time to completion of four courses of capecitabine and total dose of capecitabine. A sample size of 100 patients will be analysed between November 2016 and April 2018.

Ethics and dissemination Ethical approval was obtained at all participating institutions. The results of this study will be submitted for publication in international peer-reviewed journals.
Strengths and limitations of this study

This is the first study to analyse the safety and efficacy of cystine and theanine in patients with colorectal cancer treated with capecitabine after surgery.

- This is an adequately powered study to provide a clinically meaningful outcome.

- This study is an exploratory trial; a confirmatory trial with a larger sample size will be required subsequently.

Trial registration number UMIN000024784; Pre-results.

\section{INTRODUCTION}

In recent years, the number of deaths from colorectal cancer in Japan has been increasing remarkably. According to vital statistics in Japan, 2016, ${ }^{1}$ in women colorectal cancer is the leading cause of death above all other cancers. In men, the number of colorectal cancer deaths follows that of lung cancer and stomach cancer and the difference in the number of deaths occurring between stomach cancer and colorectal cancer has been shrinking gradually in the past several years.

In the early stages of colorectal cancer, surgical operation, including endoscopic surgical removal, is recommended, and in locally advanced cancers, combined chemotherapy after surgery is usually performed. For patients with rectal cancer, a combination of radiotherapy with chemotherapy before surgery may be a treatment option. In advanced or recurrent cancers, chemotherapy 
is the main treatment and surgery is performed only when it is possible. In addition, for clinical stage III disease, adjuvant chemotherapy may be considered. In Japan, uraciltegafur (UFT)/leucovorin (LV) is the standard adjuvant oral chemotherapy based on the National Surgical Adjuvant Breast and Bowel Project C-06 trial. ${ }^{2}$ On the other hand, in Europe and USA, 5-fluorouracil (5-FU)/LV plus oxaliplatin is the standard therapy; however, this is not the case in Japan owing to the high frequency of adverse events (AEs) associated with 5-FU/LV plus oxaliplatin, such as haematotoxicity and peripheral neuropathy, as well as the good surgical results in Japan. S-1 and capecitabine are also oral pyrimidine fluoride anticancer drugs. Recently, it was reported that adjuvant chemotherapy using S-1 for stage III colon cancer was confirmed to be non-inferior in disease-free survival rates compared with UFT $/ \mathrm{LV}{ }^{3}$ On the other hand, a randomised phase III study of adjuvant chemotherapy with S-1 versus capecitabine in patients with stage III colon cancer (JCOG0910) failed to demonstrate the non-inferiority of adjuvant S-1 compared with capecitabine treatment regarding disease-free survival. ${ }^{4}$ Furthermore, the non-inferiority of adjuvant capecitabine compared with 5-FU/LV treatment regarding disease-free survival was reported in a randomised phase III trial. ${ }^{5}$ Therefore, among the oral pyrimidine fluoride anticancer drugs, adjuvant capecitabine remains the standard treatment for stage III colon cancer.

Capecitabine, however, often causes hand-foot syndrome (HFS) as an $\mathrm{AE}^{6}{ }^{6}$ as well as gastrointestinal symptoms, such as stomatitis, anorexia, diarrhoea, bone marrow suppression and an increase in hepatobiliary enzyme levels. ${ }^{7}$ These AEs not only negatively affect treatment course but also affect a patient's quality of life (QOL). There are no standard prevention therapies for these AEs and withdrawal or a reduced dosage of capecitabine with symptomatic treatment is often required when they occur. Therefore, reduction or prevention therapy for AEs that may improve the completion rate of capecitabine treatment is considered to be useful.

\section{Cystine and theanine}

Cystine and theanine are amino acids that are known to be precursors involved in the synthesis of glutathione (GSH) in humans. GSH is vital for antioxidation reactions that reduce reactive oxygen species, such as peroxides and free radicals, and is a substrate in conjugation reactions for detoxification. Glutamine is a precursor of GSH and similarly to cystine/theanine is reported to be effective for preventing and treating oral mucositis in patients receiving mucotoxic cancer chemotherapy. ${ }^{8}$ In addition, it was reported that GSH has neuroprotective effects on oxaliplatin-based chemotherapy. ${ }^{9}$ Perioperative oral administration of cystine/theanine was also reported to suppress inflammation and enhance recovery after surgery. ${ }^{10}$

Previous research has shown that oral administration of cystine/theanine attenuates the AEs of S-1 adjuvant chemotherapy in patients with colorectal cancer after surgery. ${ }^{11}$ The incidence rate of diarrhoea was significantly less in the cystine/theanine group $(4.5 \%)$ than in the control group (41.7\%). The completion rate of the first course of S-1 adjuvant chemotherapy at the prescribed dose was significantly higher in the cystine/theanine group $(90.9 \%)$ than in the control group $(41.7 \%)$. These results of previous studies suggest that cystine/theanine will attenuate the AEs and improve the QOL of patients treated with capecitabine which is an oral pyrimidine fluoride anticancer drug similar to S-1. Therefore, we will analyse the efficacy of cystine/theanine in a randomised, double-blinded, placebo-controlled study.

\section{METHODS AND ANALYSIS \\ Design}

This study is a multi-institutional, randomised, doubleblinded, placebo-controlled, phase II trial aimed to investigate the safety and efficacy of oral administration of cystine/ theanine in patients with colorectal cancer undergoing capecitabine-based adjuvant chemotherapy after surgery (figure 1). The protocol of this study followed the Standard Protocol Items for Randomized Trials statement and its checklist (online supplementary appendix 1). ${ }^{12}$

\section{Study setting}

This study is multi-institutional with four participating institutions located in Japan, as follows: Sendai Open Hospital, Iwate Prefectural Central Hospital, Yamagata Prefectural Central Hospital and Tohoku Rosai Hospital.

\section{Patient and public involvement}

Patients and/or public were not involved.

\section{Randomisation, blinding, safety assessment and data collection}

Randomisation

Eligible patients will be enrolled in the Japanese Organisation for Research and Treatment of Cancer (JORTC) data centre using a web-based electronic data capturing system. Patients will be randomly assigned to either the cystine/theanine group or the placebo group, using stratified permuted block randomisation methods and a computer-generated randomisation schedule with a 1:1 allocation ratio, balanced for the following stratification factors: (1) study site, (2) age ( $<71$ or $\geq 71$ years) and (3) site of cancer (colon or rectum).

\section{Blinding}

Patients and investigators responsible for treatment will be blinded to the administered reagent (cystine/theanine or placebo). The study drugs will be packaged in a way in which they cannot be distinguished from one another.

\section{Safety assessment}

AEs will be graded according to the Common Terminology Criteria for AEs (CTCAE) v.4.0, Japanese Clinical 


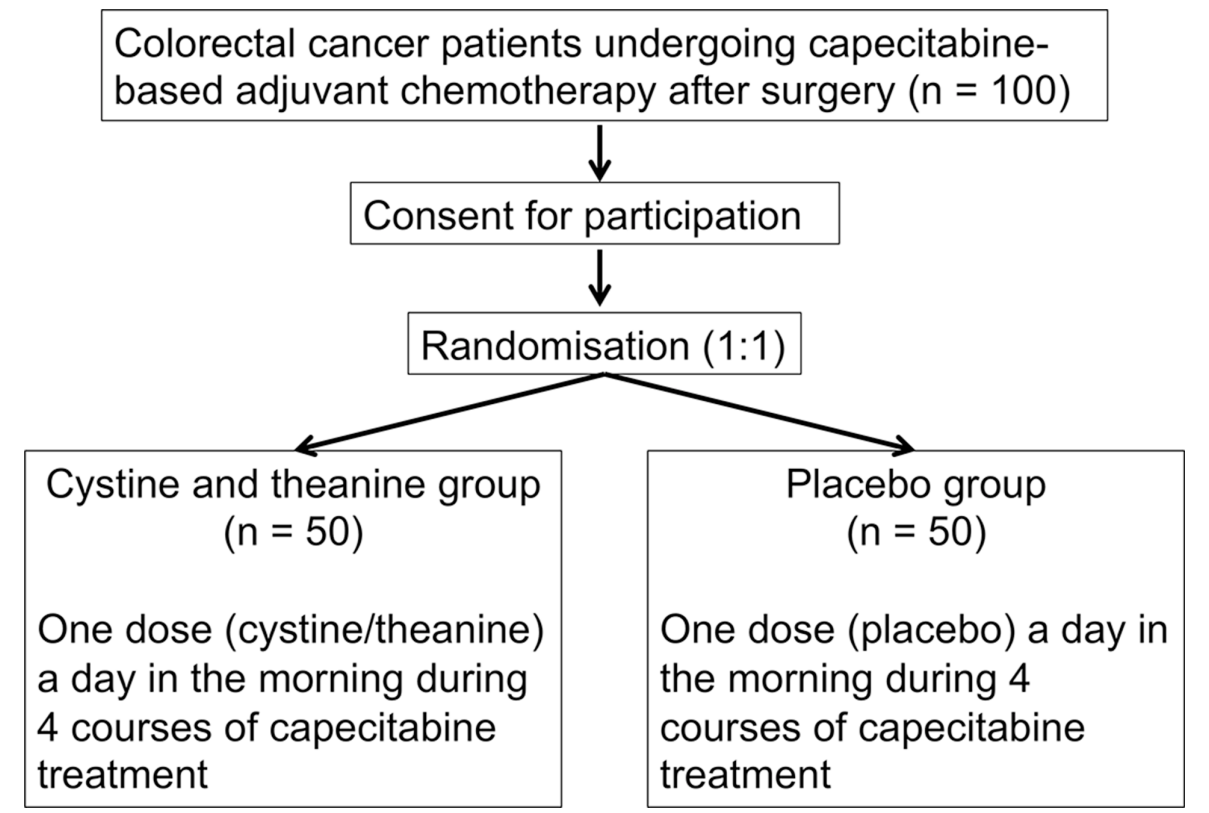

Figure 1 Flow chart of the study. Participants will be randomised (1:1 allocation ratio) into the cystine/theanine group ( $\mathrm{n}=50$ ) or the placebo group $(n=50)$.

Oncology Group (JCOG) version. HFS will be assessed by the HFS grading scale suggested by Blum et al (table 1). ${ }^{13}$

Health-related QOL assessment

Health-related QOL will be assessed using the following patient-reported outcome measures: the Japanese version of the European Organisation for Research and Treatment of Cancer (EORTC) QOL questionnaire module for all patients with cancer (QLQ-C30) and for patients with colorectal cancer (QLQ-CR29).

\section{Data collection}

We will collect basic data, such as sex, age, histology of colorectal cancer, cancer stage, daily living ability using the Eastern Cooperative Oncology Group scale of performance status (PS), height, weight and current treatment, at enrolment. Within 3 days before each capecitabine

\begin{tabular}{lll}
\hline $\begin{array}{l}\text { Table } 1 \\
\text { from }^{13} \text { ) }\end{array}$ & Hand-foot syndrome grading scale* (modified \\
\hline Grade & Clinical domaint & Functional domain \\
\hline 1 & $\begin{array}{l}\text { Numbness, dysesthesia/ } \\
\text { paresthesia, tingling, } \\
\text { painless swelling or } \\
\text { erythema. }\end{array}$ & $\begin{array}{l}\text { Discomfort that does not } \\
\text { disrupt normal activities. }\end{array}$ \\
\hline 2 & $\begin{array}{l}\text { Painful erythema with } \\
\text { swelling. }\end{array}$ & $\begin{array}{l}\text { Discomfort that affects } \\
\text { activities of daily living. }\end{array}$ \\
\hline 3 & $\begin{array}{l}\text { Moist desquamation, } \\
\text { ulceration, blistering and } \\
\text { severe pain. }\end{array}$ & $\begin{array}{l}\text { Severe discomfort, } \\
\text { unable to work or } \\
\text { perform activities of daily } \\
\text { living. }\end{array}$ \\
\hline
\end{tabular}

*This scale applies only for the grading of hand-foot syndrome and not for any other skin abnormalities and/or other cutaneous areas. †In the case of a discrepancy between clinical and functional domains, the assigned grade will be the higher of the grades. treatment course, we will analyse the weight, PS, AEs according to CTCAE v.4.0-JCOG, such as haematology (leucocytes, neutrophil counts, haemoglobin and platelets), serum chemistry (total bilirubin, alkaline phosphatase (ALP), aspartate aminotransferase (AST), alanine aminotransferase (ALT), $\gamma$-glutamyl transpeptidase $(\gamma$-GTP) and creatinine), HFS grading scale (table 1$),{ }^{13}$ QOL questionnaire (EORTC QLQ-C30 and QLQ-CR29 scores), adherence to cystine/theanine or placebo and combination treatments, until completion of protocol treatment. The data after completion (four courses) will be investigated within 3 days before the start of the fifth course of treatment. When capecitabine treatment is discontinued in the middle of a treatment course, we will collect the data within 3 days after termination of treatment.

\section{Eligibility criteria}

Eligible patients satisfying the inclusion criteria will be recruited. Inclusion and exclusion criteria are listed in box 1 .

\section{Estimated timeline}

Recruitment of study patients was initiated on 14 November 2016 and is expected to be completed by 30 April 2018. The trial is expected to be finished by 30 September 2018.

\section{Treatment}

In this study, patients with colorectal cancer who are scheduled to receive capecitabine therapy as an adjuvant chemotherapy after surgery will be administered cystine/ theanine or placebo as an intervention therapy. Capecitabine therapy will be conducted in accordance with prescribing information ${ }^{7}: 1250 \mathrm{mg} / \mathrm{m}^{2}$ twice daily orally (morning and evening; equivalent to $2500 \mathrm{mg} / \mathrm{m}^{2}$ total 


\section{Box 1 Inclusion and exclusion criteria}

\section{Inclusion criteria}

- Understanding of the nature and consequences of the study and voluntary signature of the informed consent document.

- Age $\geq 20$ and $\leq 80$ years at the time of consent.

- Histologically confirmed colorectal cancer after surgery.

- Agree to undergo adjuvant chemotherapy after surgery and plan to receive capecitabine therapy.

- Eastern Cooperative Oncology Group Performance Status $\leq 1$.

- Laboratory findings within the following ranges (at $\leq 14$ days prior to enrolment); leucocytes $\geq 3.0 \times 10^{9} / \mathrm{L}$, platelets $\geq 75 \times 10^{9} / \mathrm{L}$, haemoglobin $\geq 9.0 \mathrm{~g} / \mathrm{dL}$, total bilirubin $\leq 2.0 \mathrm{mg} / \mathrm{dL}$, aspartate aminotransferase $\leq 100 \mathrm{U} / \mathrm{L}$, alanine aminotransferase $\leq 100 \mathrm{U} / \mathrm{L}$, serum creatinine $\leq 1.5 \mathrm{mg} / \mathrm{dL}$.

\section{Exclusion criteria}

- Previous treatment with chemotherapy.

- Previous treatment with radiation.

- Currently taking dietary supplements containing amino acids or protein (allowed if discontinued for more than 7 days before the start of the study).

- Previous administration of cystine or theanine.

- Currently taking dietary supplements derived from herbs (such as St John's wort or others) except for multivitamins.

- Pregnant or breast feeding.

- Immune deficiency.

- Phenylketonuria.

- Enrolment in other clinical trials.

- Cognitive disorder prior to enrolment.

- Psychiatric disorder that may affect data sampling.

- Unable to communicate verbally.

- Incapable of oral ingestion.

- Malabsorption syndrome.

- History of gastrectomy.

- Active gastrointestinal bleeding unrelated with cancer.

Investigator considers that the patient is not suitable for the study.

daily dose) for 2 weeks followed by a 1-week rest period in 3 -week cycles for a total of eight courses (24 weeks). The dose is calculated according to body surface area (BSA) (table 2). AEs caused by capecitabine administration may be managed by dose interruptions and adjustment of capecitabine dose (table 3). The dose reduction calculations according to BSA are shown in table 4 .

\section{Interventions}

Cystine/theanine or placebo will be administered in one dose every morning from the start of capecitabine

Table 2 Capecitabine dose calculation according to body surface area

\begin{tabular}{ll}
\hline \multicolumn{2}{l}{ Dose level: $\mathbf{1 2 5 0} \mathbf{~ m g} / \mathbf{m}^{2}$ twice a day } \\
\hline Body surface area $\left(\mathbf{m}^{2}\right)$ & Total daily dose $(\mathbf{m g})$ \\
\hline$<1.33$ & 3000 (10 tablets) \\
\hline $1.33-1.56$ & 3600 (12 tablets) \\
$1.57-1.80$ & 4200 (14 tablets) \\
$\geq 1.81$ & 4800 (16 tablets) \\
\hline
\end{tabular}

Table 3 Recommended dose modifications of capecitabine

\begin{tabular}{lll}
$\begin{array}{l}\text { Toxicity } \\
\text { CTCAE grades* }\end{array}$ & $\begin{array}{l}\text { During the course } \\
\text { of therapy }\end{array}$ & $\begin{array}{l}\text { Dose adjustment } \\
\text { for next treatment } \\
\text { (\% of starting dose) }\end{array}$ \\
\hline Grade 1 & Maintain dose level & Maintain dose level \\
Grade 2 & & \\
1st appearance & Interrupt until & $100 \%$ \\
2nd appearance & $\begin{array}{l}\text { resolved to grade } \\
\text { 3rd appearance }\end{array}$ & $75 \%$ \\
4th appearance & $\begin{array}{l}\text { Discontinue } \\
\text { treatment }\end{array}$ & $50 \%$ \\
& permanently & \\
& &
\end{tabular}

Grade 3

$\begin{array}{lll}\text { 1st appearance } & \text { Interrupt until } & 75 \% \\ \text { 2nd appearance } & \begin{array}{l}\text { resolved to grade } \\ 0-1\end{array} & 50 \% \\ \text { 3rd appearance } & \begin{array}{l}\text { Discontinue } \\ \text { treatment } \\ \text { permanently }\end{array} & - \\ & \end{array}$

Grade 4

$\begin{array}{lll}\text { 1st appearance } & \text { Discontinue } & - \\ \text { treatment } & 50 \% \\ & \text { permanently or } & \\ \text { If physician decides } & \\ & \text { continuation is in } & \\ \text { the patient's best } & \\ & \text { interest, interrupt } & \\ & \text { until resolved to } \\ & \text { grade 0-1 }\end{array}$

${ }^{*}$ Common Terminology Criteria for Adverse Events v.4.0, Japanese Clinical Oncology Group version.

therapy. The intervention period will be four courses of capecitabine therapy (figure 2). Cystine/theanine or placebo administration will be continued for the entire 5-week course of capecitabine therapy, unless there is an interruption owing to AEs. Cystine/theanine or placebo

Table 4 Capecitabine dose reduction calculation according to body surface area

\begin{tabular}{llll}
\hline & \multicolumn{3}{l}{ Total daily dose $(\mathbf{m g})$} \\
\cline { 2 - 4 } Surface area $\left(\mathbf{m}^{2}\right)$ & $\mathbf{1 0 0 \%}$ & $\mathbf{7 5 \%}$ & $\mathbf{5 0 \%}$ \\
\hline$<1.13$ & $\begin{array}{l}3000(10 \\
\text { tablets })\end{array}$ & $\begin{array}{l}1800(6 \\
\text { tablets })\end{array}$ & $\begin{array}{l}1200(4 \\
\text { tablets })\end{array}$ \\
$1.13-1.20$ & & $\begin{array}{l}2400(8 \\
\text { tablets })\end{array}$ & $1800(6$ \\
$1.21-1.32$ & & & tablets $)$ \\
$1.33-1.44$ & $3600(12$ & & \\
$1.45-1.56$ & tablets $)$ & $3000(10$ & \\
$1.57-1.68$ & $4200(14$ & tablets $)$ & \\
$1.69-1.76$ & tablets $)$ & & $2400(8$ \\
$1.77-1.80$ & & $3600(12$ & tablets $)$ \\
$\geq 1.81$ & $4800(16$ & tablets $)$ & \\
& tablets) & & \\
\hline
\end{tabular}




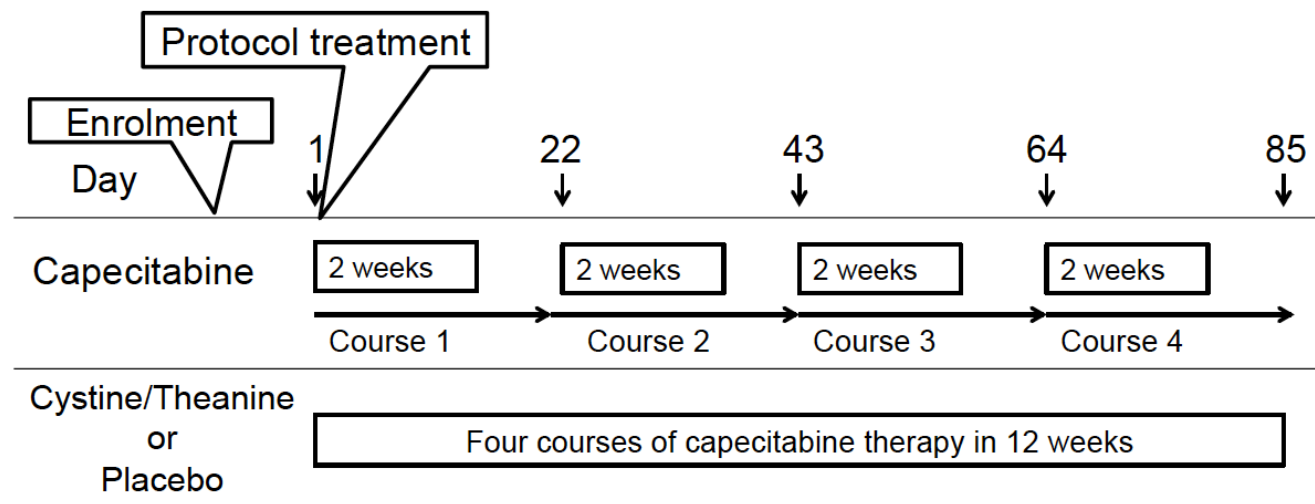

Figure 2 Schematic diagram of capecitabine and intervention treatment.

administration will be ended in accordance with the discontinuation criteria. To improve the adherence to intervention treatment, we will collect adherence records from participants at the beginning of each course (except for the first course).

\section{Cystine/theanine}

L-cystine $(700 \mathrm{mg})$, L-theanine $(270 \mathrm{mg})$ and other ingredients (maltitol, aspartame and citric acid) to a total of $1.5 \mathrm{~g}$.

\section{Placebo}

Palatinose $(833 \mathrm{mg})$, powdery starch syrup $(147 \mathrm{mg})$ and other ingredients (maltitol, aspartame and citric acid) to a total of $1.5 \mathrm{~g}$.

The amount of aspartame administered to the placebo group was $0.45 \mathrm{mg}$ less in each dose than the treatment group, to balance for the sweetness of Palatinose and powdery starch syrup.

\section{Discontinuation criteria}

Protocol treatment (cystine/theanine or placebo) will be discontinued in the following cases: (1) patient wishes to discontinue study participation, (2) recurrence of colorectal cancer, (3) complicated by another cancer, (4) CTCAE grade 3 or higher for cystine/theanine or placebo, (5) the investigator judges that it is difficult for the patient to continue the protocol treatment owing to AEs, (6) CTCAE grade 4 for capecitabine, (7) discontinuation of capecitabine treatment for longer than 3 weeks, (8) unable to continue capecitabine treatment owing to AEs, (9) patient wishes to discontinue capecitabine treatment, (10) death during protocol treatment, (11) unable to continue protocol treatment owing to a change in hospital, (12) acute progression of disease or the revelation of ineligibility or protocol violations after enrolment and (13) the investigator decides to discontinue protocol treatment due to various medical reasons.

The investigators will judge whether or not the AEs are a result of cystine/theanine or placebo. Capecitabine treatment will be continued as standard clinical therapy after discontinuation of protocol treatment based on the investigators' judgement. Dose reduction or interruption of capecitabine therapy will be considered when AEs of grade 2 or higher occur (table 3). Dose modifications of capecitabine treatment will be in accordance with the prescribing information (table 4) and will finally depend on the investigators' decision.

\section{Supportive treatment}

Dietary supplements or other drugs that contain amino acids and proteins will be prohibited because they may affect the results of the study. The concomitant use of other drugs with capecitabine will be in accordance with the prescribing information ${ }^{7}$ and the site investigators' decision. Use of other drugs and treatment concomitantly with intervention treatment will be permitted in accordance with the judgement of the site investigators. The concomitant use of drugs or treatment will be recorded regarding duration and reasons for use.

\section{Supportive treatment for HFS}

Little is known about the development of HFS and no standard prevention for HFS has been established. However, non-pharmacological interventions are recommended for the relief of symptoms, such as avoidance of pressure, prevention of injury and elevation or cooling of the hands and feet. ${ }^{14}$ Topical steroids or moisturising agents are commonly used as pharmacological interventions. Oral pyridoxine was reported to relieve symptoms of HFS, but meta-analysis failed to show any preventive effects. ${ }^{15} 16$ In addition, the COX-2 inhibitor celecoxib was reported to reduce the overall incidence of HFS. ${ }^{17} 18$

In clinical settings, moisturisation is considered to be important as a prophylactic treatment for HFS. Appropriate use of a moisturising cream may help to reduce the incidence of HFS. A randomised comparative study on prophylaxis for capecitabine-associated HFS demonstrated that a urea cream was superior to a medical ointment containing antioxidants and nourishing oil extracts (Mapisal, Medac, Wedel, Germany). ${ }^{19}$

In this trial, moisturising therapy with urea cream as a supportive treatment for HFS will be conducted from the start of protocol treatment. If the patients are taking 
other drugs or treatments for HFS, their durations and reasons for use will be recorded.

Urea ointment: Urepearl, Keratinamin, Pastaron and so on.

Directions for use: apply to hands and feet, three times a day, each time after washing hands.

\section{Study endpoints}

\section{Primary endpoint}

Primary endpoint is incidence rate of diarrhoea of grade 1 or higher (CTCAE v.4.0-JCOG).

\section{Secondary endpoints}

Secondary endpoints are EORTC QLQ-C30 and EORTC QLQ-CR29 scores; incidence rate of HFS of grade 1 or higher (HFS grading scale); incidence rate of diarrhoea of grade 2 or higher (CTCAE v.4.0-JCOG); incidence rates of AEs according to CTCAE v.4.0-JCOG of grade 1 or higher, grade 2 or higher (haematology (leucocytes, neutrophil counts, haemoglobin and platelets), serum chemistry (total bilirubin, ALP, AST, ALT, $\gamma$-GTP and creatinine), and other AEs according to CTCAE v.4.0-JCOG); adherence to protocol; completion rate of four courses of capecitabine, and the proportion of completion without delay or dose reduction; time to completion (four courses of capecitabine) and total dose of capecitabine.

\section{Statistical analysis}

\section{Study populations for analyses}

Efficacy and safety will be analysed using all randomised patients who have received at least one administration of protocol treatment. Analysis of efficacy will exclude all ineligible patients detected after randomisation, although they will be included in the analysis of safety.

\section{Analysis plan}

The primary endpoint of incidence rate of diarrhoea of grade 1 or higher (CTCAE v.4.0-JCOG) will be analysed using a one-sided Fisher's exact test at a significance level of $5 \%$. Point estimates and $90 \%$ CIs for the difference between two groups will be calculated.

\section{Sample size calculation}

A previous study showed that cystine/theanine reduced the rate of diarrhoea of S-1 adjuvant chemotherapy in patients with colorectal cancer after surgery. The incidence rate of diarrhoea was significantly less in the cystine/theanine group (4.5\%) than in the control group $(41.7 \%) .{ }^{11}$ Cystine/theanine therapy is expected to attenuate the diarrhoea of patients treated with capecitabine which is an oral pyrimidine fluoride anticancer drug similar to S-1. The incidence rate of diarrhoea after capecitabine monotherapy according to the prescribing information is $36.8 \% .^{7}$ Based on these data, we estimated that the incidence rate of diarrhoea after capecitabine monotherapy will be 5\% with cystine/theanine and $25 \%$ with placebo. Therefore, assuming a rate of exclusion of $10 \%, 50$ samples are required for each group (100 samples in total) to achieve a statistical power of
$80 \%$ with a one-sided significance level of $5 \%$. Incidence rates of AEs will be analysed using the Fisher's exact test (one-sided).

\section{Ethical aspects, informed consent and safety}

All study procedures are in accordance with the Declaration of Helsinki and the Japanese ethical guidelines for clinical research. Written informed consent from each patient will be obtained prior to enrolment (online supplementary appendix 2). An independent data monitoring committee will oversee the trial and will verify the trial procedure based on ongoing evaluation and safety data. This trial has been registered at the UMIN Clinical Trials Registry as UMIN000024784 on 10 November 2016. Modifications in the study protocol will be communicated to the institutional review board (IRB) at each participating institution as well as the independent data monitoring committee. Each ethics committee or IRB will review the informed consent materials given to the participants and adapt them in accordance with their own institutional guidelines.

\section{DISCUSSION}

Capecitabine therapy as an adjuvant chemotherapy for patients with colorectal cancer after surgery is expected to extend disease-free survival. However, capecitabine often causes AEs that affect the continuation of treatment and patients' QOL. Therefore, reduction or prevention therapy for AEs that may improve the completion rate of capecitabine treatment is required. To the best of our knowledge, there are no randomised studies on the efficacy of cystine/theanine in patients with colorectal cancer undergoing capecitabine-based adjuvant chemotherapy after surgery. In this study, we conduct a multi-institutional, randomised, doubleblinded, placebo controlled, phase II trial which is the most appropriate design to demonstrate the efficacy of a new therapy.

Cystine and theanine are amino acids contained in various foods and they are permitted as food additives and hence widely used. No severe AEs caused by cystine/ theanine have been reported in research on humans. ${ }^{20} 21$ A previous study reported that cystine/theanine attenuated the AEs of S-1 adjuvant chemotherapy in patients with colorectal cancer after surgery. ${ }^{11}$ Cystine/theanine are expected to attenuate AEs and improve the QOL of patients treated with capecitabine which is an oral pyrimidine fluoride anticancer drug, similar to S-1.

Cystine/theanine will become a possible option for reduction or prevention therapy for AEs that may occur due to capecitabine therapy in patients with colorectal cancer after surgery, and in addition, we will plan to conduct a phase III trial, if this study demonstrates the attenuation of AEs and improves the QOL of patients treated with capecitabine. 


\section{Trial status}

Enrolment started in November 2016. At the time of manuscript submission (December 2017) 52 patients have already been enrolled. We expect to finish patient recruitment by April 2018.

\section{Confidentiality}

Data will be retained in accordance with the Japanese ethical guidelines for clinical research. Participants will be allocated a unique identification (ID) number at enrolment. The master list linking personal information of the participants and ID number will be maintained in a separate locked cabinet and a password-protected hard drive at each institution. Data will be analysed by ID number only. Records will be retained for 5 years after study completion and then will be destroyed by the data centre.

\section{Ethics and dissemination}

The results of this study will be submitted for publication in international peer-reviewed journals and the key findings will be presented at conferences. Participants will be informed of the results of the trial by the investigators. Authorship will be ascribed in accordance with the International Committee of Medical Journal Editors guidelines.

\section{Author affiliations}

${ }^{1}$ Department of Palliative Medicine, The Institute of Medical Science, The University of Tokyo, Tokyo, Japan

${ }^{2}$ Department of Gastroenterological Surgery, Sendai City Medical Center Sendai Open Hospital, Sendai, Japan

${ }^{3}$ Department of Gastroenterological Surgery, Iwate Prefectural Central Hospital, Iwate, Japan

${ }^{4}$ Department of Surgery, Yamagata Prefectural Central Hospital, Yamagata, Japan ${ }^{5}$ Department of Colorectal Surgery, Tohoku Rosai Hospital, Sendai, Japan

${ }^{6}$ Japanese Organisation for Research and Treatment of Cancer (JORTC), NPO, Tokyo, Japan

Acknowledgements We are grateful for the support from Ajinomoto Co., Inc. We thank in advance all the patients, investigators and institutions involved in this study.

Contributors RH, TT, GM, TS, KT, KA, SO and SI participated in the design of the study. SO performed the statistical analysis. All authors contributed to writing and critically revising the manuscript, and all gave their final approval of the version to be published.

Funding This trial was supported by Ajinomoto Co., Inc.

Disclaimer The company did not have any role in the study design, data collection, management, analysis or interpretation of the data, writing of the manuscript or the decision to submit the manuscript for publication.

Competing interests None declared.

Patient consent Obtained.

Ethics approval Japanese Organisation for Research and Treatment of Cancer Protocol Review Committee and the Institutional Review Board at each participating institution (Sendai Open Hospital, Iwate Prefectural Central Hospital, Yamagata Prefectural Central Hospital, and Tohoku Rosai Hospital).

Provenance and peer review Not commissioned; externally peer reviewed.

Author note The JORTC Data Center and JORTC Independent Data Monitoring Committee have access to the final trial dataset. There is no contractual agreement regarding the investigators' access restrictions to the dataset.

Open access This is an open access article distributed in accordance with the Creative Commons Attribution Non Commercial (CC BY-NC 4.0) license, which permits others to distribute, remix, adapt, build upon this work non-commercially, and license their derivative works on different terms, provided the original work is properly cited, appropriate credit is given, any changes made indicated, and the use is non-commercial. See: http://creativecommons.org/licenses/by-nc/4.0/.

\section{REFERENCES}

1. Director-General for Statistics and Information Policy, Ministry of Health, Labour and Welfare. Vital statistics in Japan -Trends up to 2016.

2. Lembersky BC, Wieand HS, Petrelli NJ, et al. Oral uracil and tegafur plus leucovorin compared with intravenous fluorouracil and leucovorin in stage II and III carcinoma of the colon: results from National Surgical Adjuvant Breast and Bowel Project Protocol C-06. $J$ Clin Oncol 2006;24:2059-64.

3. Yoshida M, Ishiguro M, Ikejiri K, et al. S-1 as adjuvant chemotherapy for stage III colon cancer: a randomized phase III study (ACTS-CC trial). Ann Oncol 2014;25:1743-9.

4. Hamaguchi T, Shimada Y, Mizusawa J, et al. Randomized phase III study of adjuvant chemotherapy with S-1 versus capecitabine (cape) in patients with stage III colon cancer (CC): results of Japan Clinical Oncology Group study (JCOG0910). J Clin Oncol 2015;33. suppl; abstr 3512 .

5. Twelves C, Wong A, Nowacki MP, et al. Capecitabine as adjuvant treatment for stage III colon cancer. N Engl J Med 2005;352:2696-704.

6. Yamamoto D, Iwase S, Tsubota Y, et al. Randomized study of orally administered fluorinated pyrimidines (capecitabine versus S-1) in women with metastatic or recurrent breast cancer: Japan Breast Cancer Research Network 05 Trial. Cancer Chemother Pharmacol 2015;75:1183-9.

7. Chugai Pharmaceutical Co., Ltd. Information on proper use of capecitabine (xeloda $\AA$ ) for colorectal cancer. 2016.

8. Peterson DE, Jones JB, Petit RG. Randomized, placebo-controlled trial of Saforis for prevention and treatment of oral mucositis in breast cancer patients receiving anthracycline-based chemotherapy. Cancer 2007;109:322-31.

9. Cascinu S, Catalano V, Cordella L, et al. Neuroprotective effect of reduced glutathione on oxaliplatin-based chemotherapy in advanced colorectal cancer: a randomized, double-blind, placebo-controlled trial. J Clin Oncol 2002;20:3478-83.

10. Miyachi T, Tsuchiya T, Oyama A, et al. Perioperative oral administration of cystine and theanine enhances recovery after distal gastrectomy: a prospective randomized trial. JPEN J Parenter Enteral Nutr 2013;37:384-91.

11. Tsuchiya $\mathrm{T}$, Honda $\mathrm{H}$, Oikawa $\mathrm{M}$, et al. Oral administration of the amino acids cystine and theanine attenuates the adverse events of S-1 adjuvant chemotherapy in gastrointestinal cancer patients. Int $J$ Clin Oncol 2016;21:1085-90.

12. Chan AW, Tetzlaff JM, Gøtzsche PC, et al. SPIRIT 2013 explanation and elaboration: guidance for protocols of clinical trials. BMJ 2013;346:e7586.

13. Blum JL, Jones SE, Buzdar AU, et al. Multicenter phase II study of capecitabine in paclitaxel-refractory metastatic breast cancer. $J$ Clin Oncol 1999;17:485-93.

14. Ministry of Health, Labour and Welfare. Manual for handling disorders due to adverse drug reactions: hand-foot syndrome. 2010.

15. Nagore E, Insa A, Sanmartín O. Antineoplastic therapy-induced palmar plantar erythrodysesthesia ('hand-foot') syndrome. Incidence, recognition and management. Am J Clin Dermatol 2000;1:225-34.

16. Zhou Y, Peng L, Li Y, et al. Prophylactic pyridoxine was not able to reduce the incidence of capecitabine-induced hand-foot syndrome: A meta-analysis. Biomed Rep 2013;1:873-8.

17. Zhang RX, Wu XJ, Lu SX, Xj W, Sx L, et al. The effect of COX-2 inhibitor on capecitabine-induced hand-foot syndrome in patients with stage II/III colorectal cancer: a phase II randomized prospective study. J Cancer Res Clin Oncol 2011;137:953-7.

18. Zhang RX, Wu XJ, Wan DS, et al. Celecoxib can prevent capecitabine-related hand-foot syndrome in stage II and III colorectal cancer patients: result of a single-center, prospective randomized phase III trial. Ann Oncol 2012;23:1348-53.

19. Hofheinz RD, Gencer D, Schulz H, et al. Mapisal versus urea cream as prophylaxis for capecitabine-associated hand-foot syndrome: a randomized phase iii trial of the AIO Quality of Life Working Group. J Clin Oncol 2015;33:2444-9.

20. Miyagawa K, Hayashi Y, Kurihara S, et al. Co-administration of I-cystine and I-theanine enhances efficacy of influenza vaccination in elderly persons: nutritional status-dependent immunogenicity. Geriatr Gerontol Int 2008;8:243-50.

21. Kurihara S, Yoshida S, Sukegawa E, et al. Evaluation of safety of long-term and excessive intake of I-cystine and I-theanine in healthy adult subjects. Seikatsu Eisei 2008;52:229-36. 